\title{
Correction: PLA2G6-associated neurodegeneration presenting as a complicated form of hereditary spastic paraplegia
}

\author{
Kishin Koh ${ }^{1}$ Y Yuta Ichinose ${ }^{1}$ Hiroyuki Ishiura ${ }^{2} \cdot$ Haitian Nan $^{1} \cdot$ Jun Mitsui ${ }^{3}$ - Junya Takahashi ${ }^{4}$ Wakiro Sato ${ }^{5}$. \\ Yoshiaki Itoh $^{6} \cdot$ Kyoko Hoshino $^{7}$ - Shoji Tsuji ${ }^{3,8} \cdot$ Yoshihisa Takiyama ${ }^{1}$ Japan Spastic Paraplegia Research \\ Consotium
}

Published online: 9 November 2018

(c) The Author(s) under exclusive licence to The Japan Society of Human Genetics 2018

Correction to: Journal of Human Genetics https://doi.org/ 10.1038/s10038-018-0519-7; published online 09 October 2018

The originally published version of this article contained an error in Fig. 1 and Table 2. The correct figure and table of this article should have read as below. This has now been corrected in the PDF and HTML versions of the article. The authors apologize for any inconvenience caused.
Yoshihisa Takiyama

ytakiyama@yamanashi.ac.jp

1 Department of Neurology, Interdisciplinary Graduate School of Medicine and Engineering, University of Yamanashi, Yamanashi, Japan

2 Department of Neurology, Graduate School of Medicine, The University of Tokyo, Tokyo, Japan

3 Department of Molecular Neurology, The University of Tokyo, Tokyo, Japan

4 Department of Pediatrics, National Mie Hospital, Mie, Japan

5 Department of Immunology, National Institute of Neuroscience, National Center of Neurology and Psychiatry, Tokyo, Japan

6 Department of Neurology, Osaka City University, Osaka, Japan

7 Department of Pediatrics, Minamiwakayama Medical Center, Wakayama, Japan

8 International University of Health and Welfare Graduate School, Chiba, Japan 


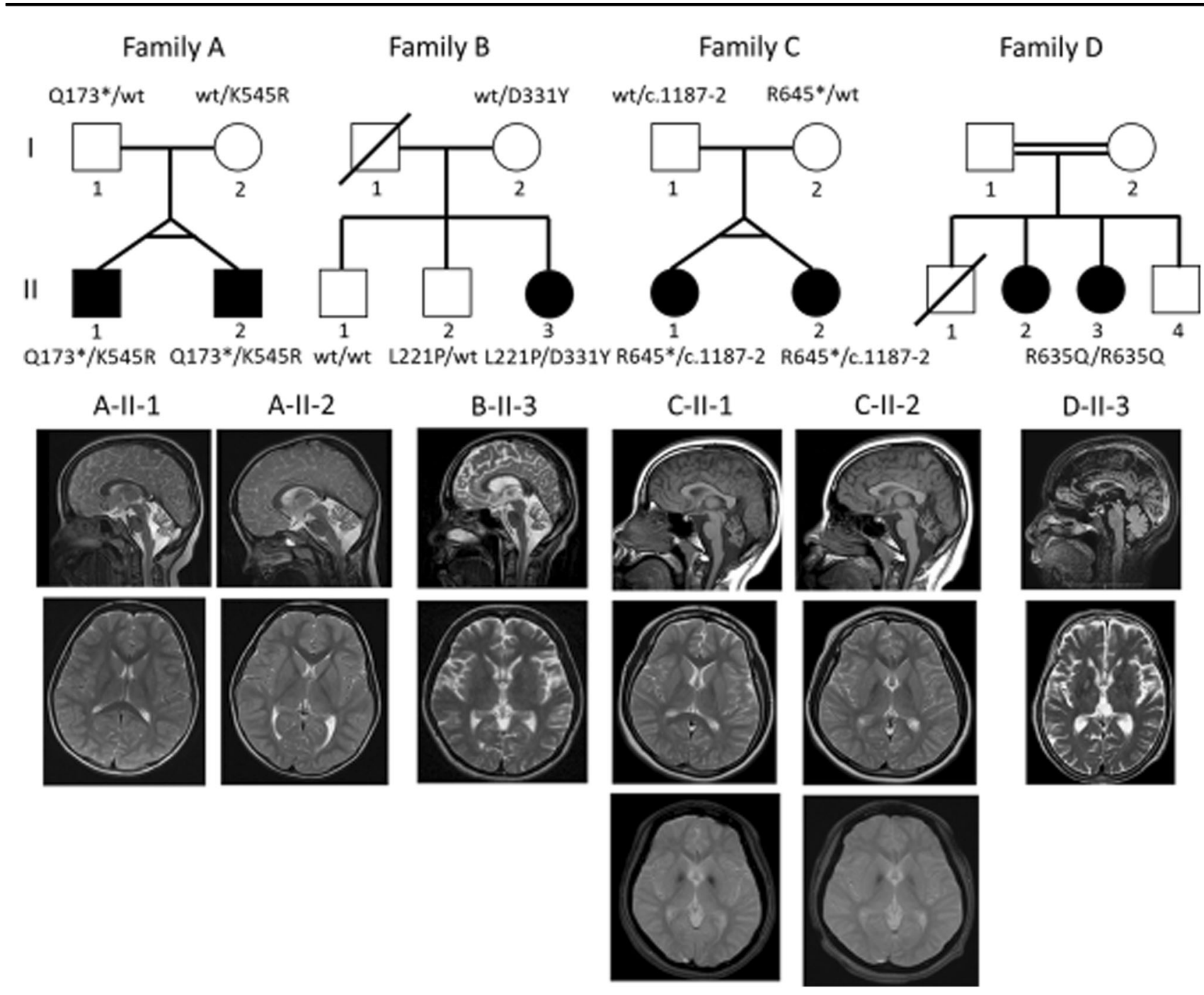

Fig. 1 
Table 2

\begin{tabular}{lllllll}
\hline Patient & A-II-1 & A-II-2 & B-II-3 & C-II-1 & C-II-2 & D-II-3 \\
\hline Age of onset (y.o.) & Infantile & Infantile & 10 & 1 & 1 & 66 \\
Gender & Male & Male & Female & Female & Female & Female \\
Initial symptoms & Gait & Gait & Gait & Mental & Mental & Gait \\
& impairment & impairment & impairment & retardation & retardation & impairment \\
Pyramidal signs & + & + & + & + & + & + \\
Cerebellar ataxia & + & + & + & - & - & - \\
Extrapyramidal & - & - & + & - & - & + \\
signs & & & & & & - \\
Mental retardation & + & + & + & + & + & - \\
Cerebellar atrophy & + & + & + & + & + & 2 \\
on brain MRI & & 4 & 5 & 4 & 4 & \\
$\begin{array}{l}\text { Modified rankin } \\
\text { scale }\end{array}$ & 4 & & & & & \\
\hline
\end{tabular}

